\title{
Claudia Bieling
}

Heimat, Inspiration, Erholung: Wie Landschaften auf immaterielle Weise zur Lebensqualität beitragen

In: Berichte und Abhandlungen / Berlin-Brandenburgische Akademie der Wissenschaften

(vormals Preußische Akademie der Wissenschaften) ; 16.2014, S. 17-25 


\section{Heimat, Inspiration, Erholung: Wie Landschaften auf immaterielle Weise zur Lebensqualität beitragen}

Dieser Beitrag behandelt einen bestimmten Teilbereich von Ökosystemleistungen nämlich die physisch nicht greifbaren, also immateriellen Formen des Nutzens, die Menschen aus ihrer natürlichen Umgebung ziehen. In der Sprache des Konzepts Ökosystemleistungen werden sie als kulturelle Ökosystemleistungen bezeichnet. Zunächst werde ich einen Überblick darüber vermitteln, welche verschiedenen Formen immateriellen Nutzens es gibt. Um möglichst konkrete Bilder und Eindrücke zu vermitteln, beziehe ich mich auf das Beispielgebiet Schwäbische Alb, wo meine Forschung hauptsächlich angesiedelt ist, und stelle dabei zwei verschiedene Annäherungsformen an kulturelle Ökosystemleistungen vor. In einem zweiten Teil werden die Verbindungen zwischen kulturellen Ökosystemleistungen und Lebensqualität behandelt. Dies mündet schließlich in einem Fazit zur Bedeutung kultureller Ökosystemleistungen im Kontext mitteleuropäischer Kulturlandschaften.

\section{Annäherungen an kulturelle Ökosystemleistungen}

Kulturelle Ökosystemleistungen bezeichnen alle Formen des immateriellen Nutzens, der Menschen aus ihrer natürlichen Umgebung entsteht (MA 2005). Darunter fallen verschiedene, nicht immer ganz scharf voneinander abzugrenzende Aspekte:

- Erholung

- ästhetische Werte

- Inspiration (vor allem für kreatives Schaffen, das auf vielerlei Arten seinen Ausdruck finden kann, z. B. in Bildern, Texten oder auch in Alltagsgegenständen)

- spirituelle und religiöse Werte

- historische Werte (im Hinblick auf ein über die Einzelperson hinaus bedeutsames kulturelles Erbe oder im Hinblick auf die individuelle Familiengeschichte, die sich mit einem Stück Land verbinden kann)

- Identität (z. B. Landschaften als Heimat, aus der viele Menschen eine tiefe Befriedigung ziehen) 
Dieses breite Spektrum stellt wie hier abgebildet sicher nur ein Grobgerüst dar man könnte anders untergliedern oder etwas hinzufügen. Klarheit besteht jedoch dahingehend, dass es sehr schwierig ist, diese immateriellen Formen des Nutzens näher zu bestimmen, also über diese Schlagworte hinaus zu beschreiben. Das betrifft die Wissenschaft - so gibt es im „Millennium Ecosystem Assessment“ (MA 2005) wie in einer Reihe von anderen Studien große Lücken zu kulturellen Ökosystemleistungen, weil man keine Daten dazu erheben konnte. Allenfalls zur Erholungsnutzung und dort vor allem zum Tourismus gibt es noch eine gute Datengrundlage. Aber auch abseits der Forschung ist für jeden leicht nachzuvollziehen, wie schwierig es ist, sich über Verbindungen von Landschaft zur eigenen Identität oder zu religiösen Werten klar zu werden oder sie gar in Worte zu fassen.

Dennoch möchte ich im Folgenden versuchen, die Schlagworte „Identität“ und „Kulturerbe“ auszufüllen und nähere Eindrücke zu vermitteln, was immaterielle Formen des Nutzens aus Kulturlandschaften genau umfassen können. Dabei beziehe ich mich auf das Beispiel der Schwäbischen Alb im Südwesten Deutschlands.

\section{Annäherung 1: Zeichen immaterieller Nutzungen}

Ich habe zunächst versucht, mich der Frage „Welches sind die immateriellen Formen des Nutzens der Schwäbischen Alb?"“ auf eine Art und Weise anzunähern, die naiv erscheinen mag: Ich habe mich auf die Suche nach sichtbaren Zeichen solcher Nutzungen gemacht. Dazu bin ich ein beispielhaft ausgewähltes Gebiet systematisch abgelaufen und habe alles erfasst, was davon zeugt, dass hier Menschen einen nicht physisch greifbaren Nutzen von der Landschaft haben. Ich nehme sie jetzt mit auf einen kleinen Spaziergang durch ein Tal, das durch Streuobstwiesen geprägt ist, und zeige Ihnen einige Bilder von dem, was mir aufgefallen ist:

Viele Sitzbänke (Abb. 1), teils mit Tischen, zeugen davon, dass sich hier Leute erholen, sicherlich die Schönheit der Landschaft genießen oder sich vielleicht auch inspirieren lassen.

Mir sind einige Einrichtungen aufgefallen, die zur Erholung oder auch zum Spiel genutzt werden - eine Strickleiter, die an einem Baum hängt, eine Lagerfeuerstelle oder kleine Hütten.

Viele Wege, die ausschließlich zum Wandern, Spazierengehen und so weiterdienen, zeugen von Aktivitäten, die Menschen zu einem immateriellen Nutzen ausüben, genauso wie Wegmarkierungen oder Hinweisschilder. Da kann es um die Erholung beim Mountainbiken gehen, um die schöne Aussicht etwa oder auch um die Auseinandersetzung mit einem Kulturerbe, nämlich die Besichtigung einer alten Burg.

Kleine Gärten zur Selbstversorgung (Abb. 2) und Hochsitze für die Jagd - hier mag sich der Zusammenhang mit immateriellen Werten vielleicht nicht auf den ersten Blick erschließen. Bei den Gärten könnte man sagen: Hier geht es doch um eine Versor- 


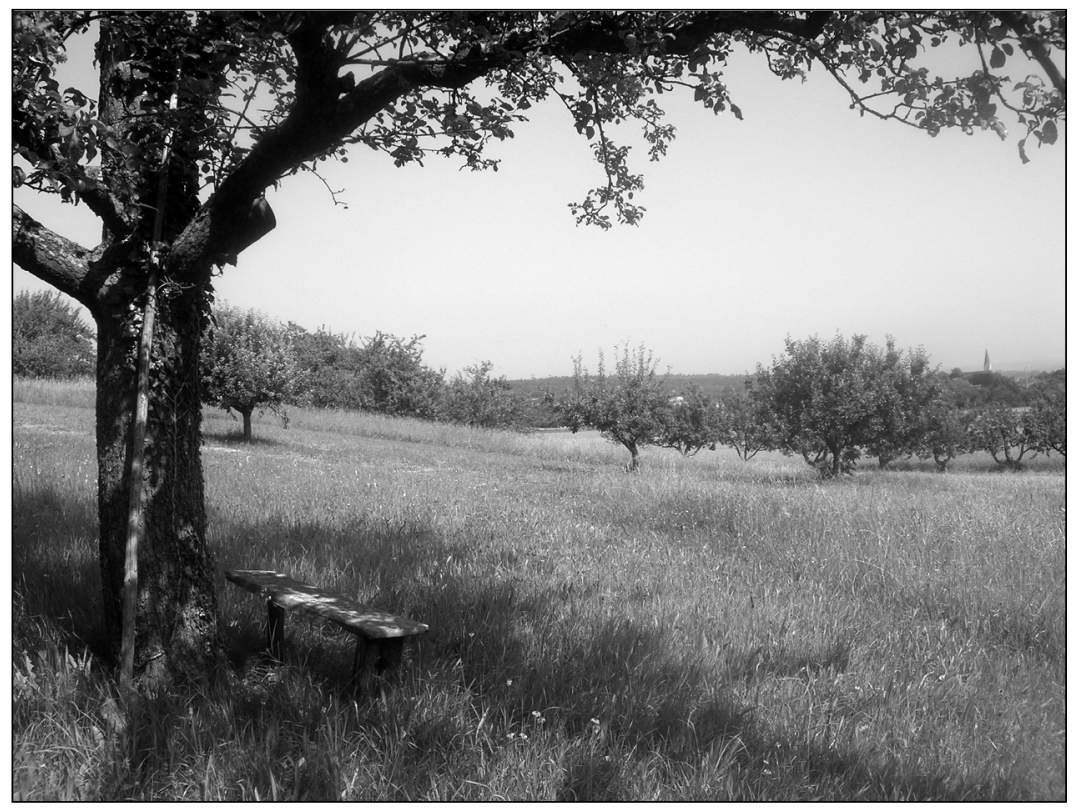

Abbildung 1

Sitzbank im Lenninger Tal, Schwäbische Alb (Foto: Claudia Bieling)

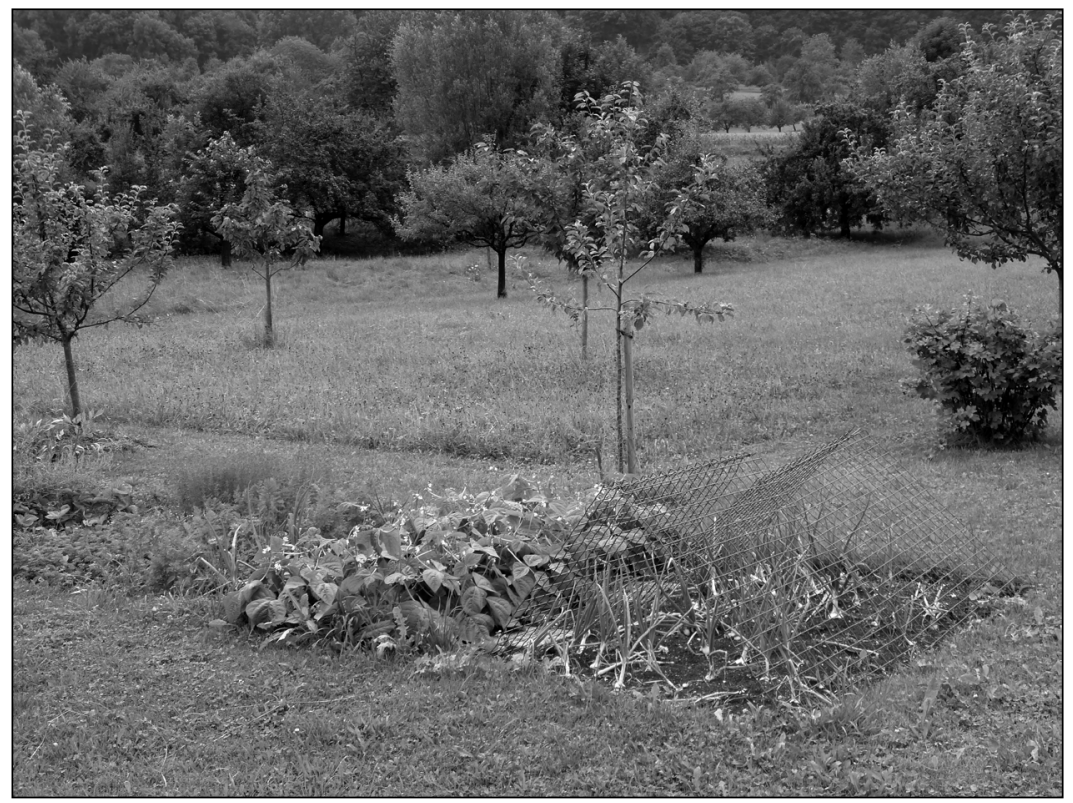

Abbildung 2

Kleingarten in den Streuobstwiesen des Lenninger Tals, Schwäbische Alb (Foto: Claudia Bieling) 
gungsleistung, der Nutzen ist der Salat oder der Lauch. Jedoch treffen die 10 Salatköpfe und das Kilo Lauch allein nicht den Kern der Sache. Es geht vielmehr darum, dass der Salat hier und mit eigenen Händen angebaut wurde, dass man den Kindern zeigen konnte, wie er wächst, vielleicht auch, dass man diese besondere Sorte anbauen konnte, die man vom Nachbarn oder der Oma bekommen hat. Die reine Versorgungsleistung würde man viel einfacher und billiger in jedem Laden bekommen. Gärtnern hat eine enge Verbindung zur Identität, hier findet ein bestimmter Lebensstil einen Ausdruck, bestimmte Werte, die man vertritt. Und auch für die Jagd, die ja zumeist von Hobbyjägern ausgeübt wird, ist bekannt, dass diese Tätigkeit viel darüber aussagt, wie man sich selbst sieht und wie man auch von anderen gesehen werden möchte - und das ist oft wichtiger als das Stück Wild, das man gelegentlich mit nach Hause bringt.

Auch Zeugnisse historischer Werte konnte ich identifizieren: Zum Beispiel die gut erhaltene und aufwendig mit Informationstafeln ausgestattete Burg, die über ihre spezifische Lage eng an naturräumliche Faktoren gebunden ist und mit der Zeit eine kulturhistorische Bedeutung entwickelte. Aber auch Beispiele für individuelle Erinnerungen waren zu finden, wie etwa die Gedächtnisplakette an einer Bank (Abb. 3) oder ein Herz, das in die Rinde eines Baumes geschnitten wurde.

Das Fazit dieser Annäherungsform an kulturelle Ökosystemleistungen (vgl. Bieling \& Plieninger 2012): Auf den untersuchten 19 Hektar Streuobstwiese gab es eine Vielzahl sichtbarer Dinge, die auf immaterielle Formen des Nutzens hinweisen wenn man mit offenen Augen durch die Landschaft geht, erschließt sich tatsächlich einiges, was „kulturelle Ökosystemleistungen“ vor Ort konkret ausmacht. In diesem Beispielgebiet gab es insbesondere viele Zeugnisse für Erholungswerte sowie für Verbindungen zu Identität, in geringerem Umfang auch für Werte im Hinblick auf Ästhetik und Kulturerbe.

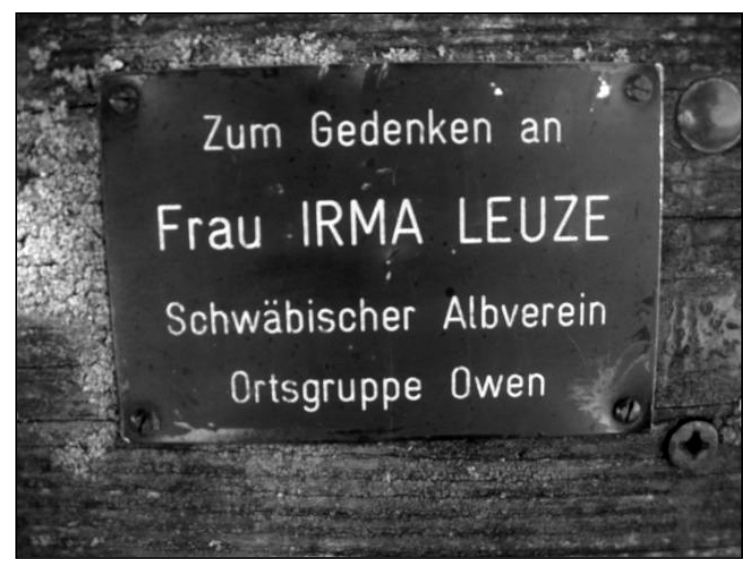

Abbildung 3

Gedächtnisplakette an einer Bank im Lenninger Tal, Schwäbische Alb (Foto: Claudia Bieling) 


\section{Annäherung 2: Kurzgeschichten}

Einen zweiter Versuch, die Frage „Was sind kulturelle Ökosystemleistungen der Schwäbischen Alb?" zu beantworten, stellt die Analyse von Kurzgeschichten dar, die Menschen über ihre Region geschrieben haben. Diese Geschichten entstanden im Winter 2010/2011 im Rahmen eines Wettbewerbs, den die Verwaltung des Biosphärengebiets Schwäbische Alb ausgeschrieben hatte. Unter dem Motto „BIOSPHÄRE - BeSchreib uns Deine Alb“ wurde die örtliche Bevölkerung aufgefordert, Geschichten zu vier Fragen einzureichen:

- Was ist für Sie das Besondere der Schwäbischen Alb (heute, gestern, morgen)?

- Was sind besondere Orte?

- Wie sind die Menschen der Schwäbischen Alb?

- Wie stellen Sie sich das Leben in der Biosphäre Schwäbische Alb im Jahr 2020 vor?

Es handelte sich also um sehr offene Fragen, und die Teilnehmer konnten ganz frei in Worte fassen, was sie mit der Schwäbischen Alb verbinden - Werte, Wünsche, Visionen. Und das sind die Ergebnisse: 42 Geschichten wurden eingereicht. Bis auf drei behandeln alle Geschichten immaterielle Formen des Nutzens, also kulturelle Ökosystemleistungen, und die meisten handeln ausschließlich davon. In den Geschichten spiegeln sich besonders Bezüge zur persönlichen Identität - mehr als drei Viertel der Geschichten sprechen diesen Aspekt an. Aber auch Erholung, Ästhetik und Kulturerbe sind besonders wichtige Themen.

Als Beispiel für das Thema „Identität“ möchte ich auf die Geschichte „Der Weg nach oben" von Johannes Stockmayer näher eingehen. Der Autor beschreibt eine Wanderung aus dem Tal auf die Hochfläche der Alb.

Noch unten im Tal stellt er fest:

„Im Augenblick sind meine Gedanken noch wirr. Beim Gehen ordnen sie sich und finden ihre Bedeutung.“

Oben angekommen heißt es dann:

„Ich schaue hinab und sehe das Land unter mir aus einer anderen Perspektive. Wo ich her komme, was hinter mir liegt, ist winzig klein und vielfältig, das Meinige ist nur ein kleiner Teil des großen Ganzen.“

Schließlich muss er den Rückweg antreten, aber etwas bleibt:

„Mein Weg endet, wo der Bach im Untergrund verschwindet. Aber in meinem Herzen ist Weite.“ 
Wie diese kurzen Textausschnitte nur anreißen - die ganze Geschichte stellt ein ständiges Widerspiegeln von der wahrgenommenen Landschaft und der inneren Befindlichkeit, von Werten und Sinnsuche dar. Hier drückt sich eine enge Wechselwirkung zwischen Landschaft und menschlicher Identität aus, ein gegenseitiges Aufeinander-Bezogensein.

Einige weitere Beispiele (ohne direkte Zitate): So stellt eine Geschichte dar, wie eine Sitzbank, das „Rietheimer Bänkle“, über Jahrzehnte hinweg Treffpunkt für mehrere Generationen einer Familie und ihrer Freunde ist und so eine Art Dreh- und Angelpunkt der Familiengeschichte ausmacht. Eine andere Geschichte beschreibt sehr eindrücklich, wie die Schönheit der Landschaft für die Autorin als Quelle kreativer Schaffenskraft wirkt. Und schließlich möchte ich noch eine Geschichte zum Thema Heimat erwähnen: Dort wird, anhand der Lebensgeschichte von einem Mann, in Worte gefasst, wie es zu Glück und geistiger Gesundheit beiträgt, wenn man an dem als Heimat empfundenen Ort leben kann beziehungsweise zu diesem zurückkehrt.

Mit diesen hoffentlich bereichernden Eindrücken möchte ich den ersten Teil des Beitrags schließen und zu der Frage übergehen, wie Lebensqualität mit kulturellen Ökosystemleistungen zusammenhängt.

\section{Verbindungen zwischen kulturellen Ökosystemleistungen und Lebensqualität}

Nach den schönen Bildern und Geschichten wird es nun etwas theoretischer - zu diesem Thema möchte ich nämlich auf ein Konzept zurückgreifen, das im Rahmen des „Millennium Ecosystem Assessment“ (MA 2005) erarbeitet wurde (Abb. 4). Hier werden Aussagen dazu gemacht, wie stark sich die verschiedenen Formen von Ökosystemleistungen auf Lebensqualität auswirken. Für die Lebensqualität unterscheidet diese Studie verschiedenen Aspekte: Sicherheit, materielle Grundversorgung, Gesundheit, gute soziale Beziehungen und schließlich, gewissermaßen durch diese vier Bereiche bedingt, die Wahl- und Handlungsfreiheit.

Wie die verschiedenen Ökosystemleistungen sich auf diese Aspekte von Lebensqualität auswirken wird durch die Dicke der Pfeile ausgedrückt - je dicker der Pfeil, desto stärker die Verbindung. Wir sehen zum Beispiel, sehr einleuchtend, eine starke Verbindung zwischen den Versorgungsleistungen und der materiellen Grundversorgung. Für die kulturellen Leistungen sieht das „Millennium Ecosystem Assessment“ nur schwache Verbindungen zur Lebensqualität, am stärksten ist noch der Beitrag zu den sozialen Beziehungen. Hierzu möchte ich in Erinnerung rufen: Innerhalb der Studie ist es nur ansatzweise gelungen, die kulturellen Ökosystemleistungen zu erfassen. Entsprechend lückenhaft ist die Datenbasis für alle weiteren Auswertungen, 


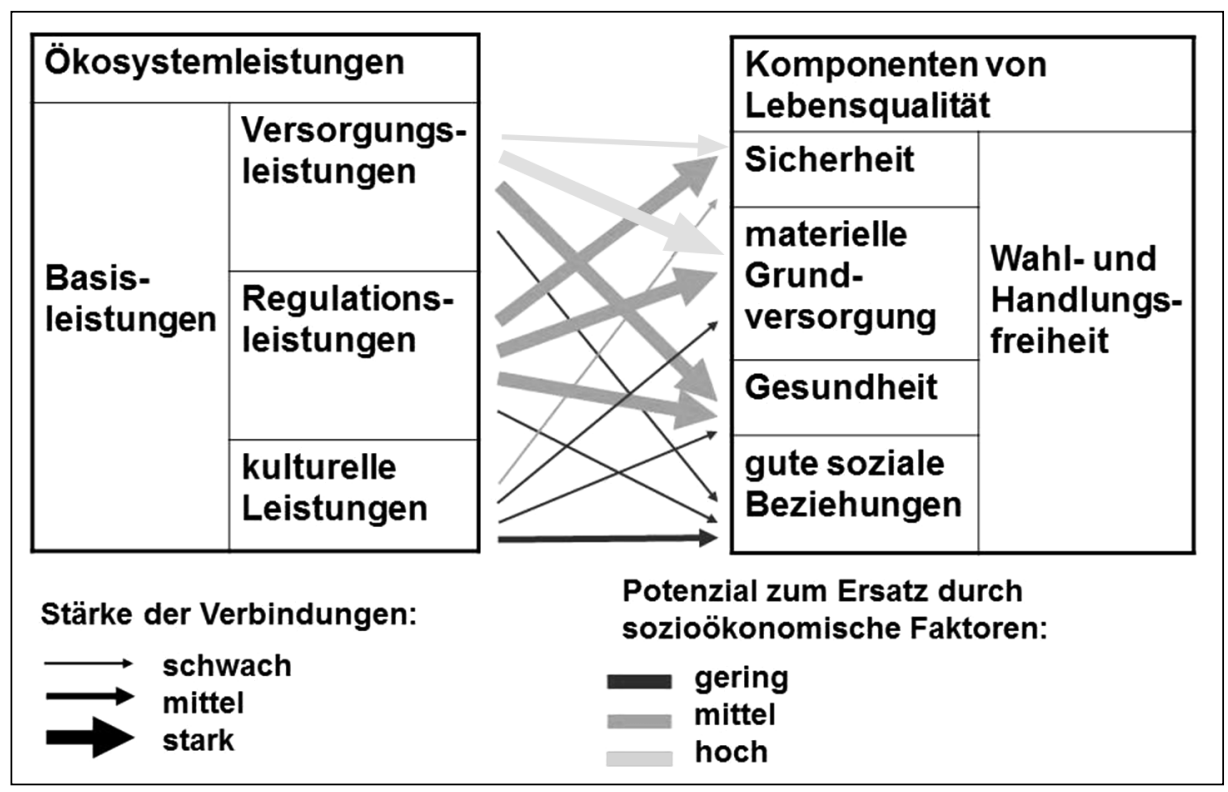

Abbildung 4

Zusammenhänge zwischen Ökosystemleistungen und Lebensqualität nach dem Millennium Ecosystem Assessment (Quelle: übersetzt und verändert nach MA 2005: vi)

und so auch für die Beiträge zur Lebensqualität. Hier dürften sich also eher methodische Probleme widerspiegeln als eine wirklich umfassende Abbildung der $\mathrm{Zu}$ sammenhänge - so hoffe ich doch auch, dass die Bilder und Geschichten von der Schwäbischen Alb verdeutlicht haben, dass immaterielle Leistungen ganz erheblich zum Wohlfinden beitragen.

Sehr bedenkenswert ist aber eine weitere Aussage, die das „Millennium Ecosystem Assessment" anhand dieses Schemas trifft. Es beleuchtet nämlich die Frage, ob es sich zwangsläufig in einer geringeren Lebensqualität äußert, wenn eine Ökosystemleistung zurückgeht. Fällt eine Versorgungsleistung oder eine kulturelle Leistung aus - zieht das automatisch eine geringere Lebensqualität nach sich oder ist es möglich, das zu kompensieren? Hierzu stellt das „Millennium Ecosystem Assessment“ fest: Manche Ökosystemleistungen sind sehr gut ersetzbar, zumindest, wenn man über Geld verfügt, über Institutionen wie funktionierende Märkte, über Technologien und Wissen. Zum Beispiel: Ist in einem Gebiet der Wald gerodet, kann man Holz aus einem anderen Gebiet kaufen - wenn ein entsprechender Markt für Holz vorhanden ist, man das Geld hat, über die Transportmöglichkeiten verfügt und so weiter.

Für die Versorgung mit Holz ist also unter bestimmten Umständen eine Ersetzbarkeit gegeben, was nicht unbedingt für andere Ökosystemleistungen zutrifft. Wie stark Leistungen über sozioökonomische Faktoren kompensiert werden können, sehen 
wir in Abbildung 4 an der Farbintensität der Pfeile - am hellsten sind die relativ gut ersetzbaren Verbindungen, vor allem bei den Versorgungs- und Regulationsleistungen. Für die kulturellen Leistungen ist jedoch eine fehlende oder kaum vorhandene Ersetzbarkeit vermerkt. Ein bestimmtes Kulturerbe, spirituelle Werte oder das Heimatempfinden sind zwingend an ganz bestimmte Orte gebunden. Diese fehlende Ersetzbarkeit betrifft fast alle immateriellen Formen des Nutzens - bis auf den Bereich Erholung: Zumindest Touristen haben die Wahl, sich ein anderes Urlaubsgebiet zu suchen, wenn das bisherige nicht mehr genutzt werden kann. Generell bleibt jedoch festzuhalten: Verminderte kulturelle Leistungen können über den Einsatz von Geld, Technik und so weiter in der Regel nicht abgemildert werden; sie gehen recht sicher mit einer verminderten Lebensqualität einher.

Dies spiegelt sich auch in einer anderen Studie wider. Guo et al. (2010) haben untersucht, inwiefern Ökosystemleistungen und wirtschaftliche Entwicklung zusammenhängen. Ganz plakativ formuliert: Kann man sich von den negativen Auswirkungen der Degradation von Ökosystemleistungen „,freikaufen“? Geht also ein hoher ökonomischer Standard einher mit einer geringeren Abhängigkeit von Ökosystemleistungen? Dazu wurden Daten für rund 150 Länder statistisch analysiert. Dabei zeigt sich, dass Menschen im Zuge der wirtschaftlichen Entwicklung insgesamt abhängiger von ihrer natürlichen Lebensgrundlage werden. Es ist jedoch zu unterscheiden: Versorgungsleistungen werden weniger bedeutsam, für Regulierungsleistungen und vor allem kulturelle Leistungen zeigt sich jedoch eine steigende Abhängigkeit. Auch hier findet sich also ein Hinweis auf die fehlende Ersetzbarkeit von immateriellen Formen des Nutzens.

Kurz vor dem Ende meines Beitrags möchte ich noch einen Ausblick unternehmen: Welche Rolle spielen kulturelle Ökosystemleistungen bei Entscheidungen zur Landnutzung, also/gerade für die Landeigentümer, die durch ihre Bewirtschaftung das Bild unsere Landschaften maßgeblich prägen? Studien zeigen, dass immaterielle Werte eine große Rolle spielen, trotz aller ökonomischen Zwänge. Zum Beispiel habe ich selbst eine Studie durchgeführt, wo es um die die Frage ging, wie Waldbauern sogenannten naturnahen Bewirtschaftungsformen gegenüberstehen (Bieling 2004). Die Akzeptanz solcher Ansätze hängt für eine Mehrzahl der befragten Personen weniger von Förderprogrammen und anderen finanziellen Anreizen ab, sondern entscheidend davon, ob diese Bewirtschaftungsformen ins jeweilige Weltbild, Wertesystem oder zu den Familientraditionen passen. Nicht zuletzt: Viele von Naturschutzseite heute „erwünschte“ Landnutzungsformen wie Streuobstanbau oder die Pflege von Hecken sind völlig unrentabel und wären schon längst aufgegeben worden, wenn Landeigentümer sich nicht ideellen Werten verpflichtet fühlen würden. Aber auch mit Blick auf die Gesellschaft insgesamt wage ich festzustellen: Wir sehen hier eine wachsende Beachtung von immateriellen Werten, insbesondere von Erho- 
lungswert, Landschaftsästhetik und Erhaltung von Kulturerbe - in politischen Maßnahmen, vor allem aber auf der Ebene der immer zahlreicheren lokalen Initiativen, die sich der Kulturlandschaftserhaltung annehmen.

\section{Fazit}

Kulturelle Ökosystemleistungen spielen in mitteleuropäischen Kulturlandschaften eine zentrale Rolle:

Es gibt vielfältige Formen des immateriellen Nutzens aus Landschaften, und diese kulturellen Leistungen tragen zur Lebensqualität bei.

Kulturelle Leistungen sind nicht oder kaum ersetzbar - mit unserem gehobenen wirtschaftlichen Standard können wir zwar Agrarprodukte aus anderen Regionen der Welt importieren. Aber Werte wie Naherholung, Heimat oder unsere historischen Wurzeln sind untrennbar mit den hiesigen Kulturlandschaften verknüpft - wenn wir sie hier nicht erhalten, sind sie verloren.

Schließlich sind kulturelle Leistungen ein wichtiges Motiv in der Landnutzung. Entsprechend können sie als Argumente, als Hebel, eingesetzt werden, um auf die Erhaltung solch reicher Kulturlandschaften hinzuwirken. Dies knüpft an ein wichtiges Motiv für Landeigentümer an. Aber auch die Gesellschaft insgesamt dürfte mit Verweis auf die kulturellen Leistungen durchaus zu öffentlichen Investitionen in die Landschaftserhaltung bereit sein.

\section{Literatur}

Bieling, C. (2004): Non-industrial private-forest owners: possibilities for increasing adoption of close-to-nature forest management. In: European Journal of Forest Research 123, 4, S. 293-303.

Bieling, C. \& T. Plieninger (2014): Recording manifestations of cultural ecosystem services in the landscape. In: Landscape Research 38, 5, S. 649-667.

Guo, Z., Zhang, L. \& Li, Y. (2010): Increased dependence of humans on ecosystem services and biodiversity. In: PLoS ONE 5, 10, e13113.

MA (Millennium Ecosystem Assessment) (2005): Ecosystems and Human Well-Being: Synthesis. Washington, D.C. 